\title{
An efficient scoring model for diagnosis of tuberculous pleural effusion
}

\section{Senquan Wu ( $\sim 873508640 @ q q . c o m$ )}

Dongguan People's Hospital

\section{Shaomei Li}

Dongguan People's Hospital

Nianxin Fang

Dongguan People's Hospital

\section{Weiliang Mo}

Dongguan People's Hospital

Huadong Wang

Jinan University

Ping Zhang

Dongguan People's Hospital

\section{Research Article}

Keywords: Tuberculosis, Pleural effusion, Tuberculous pleural effusion, Scoring model

Posted Date: October 8th, 2021

DOI: https://doi.org/10.21203/rs.3.rs-957673/v1

License: (c) (i) This work is licensed under a Creative Commons Attribution 4.0 International License.

Read Full License 


\section{An efficient scoring model for diagnosis of tuberculous pleural effusion}

Senquan $\mathrm{Wu}^{1,2^{*}}$, Shaomei $\mathrm{Li}^{1 \#}$, Nianxin Fang ${ }^{1 \#}$, Weiliang Mo ${ }^{1}$, Huadong Wang $^{2^{*}}$ and Ping Zhang ${ }^{1^{*}}$

${ }^{I}$ Department of Respiratory and Critical Care Medicine, Dongguan People's Hospital, Dongguan, Guangdong, China; ${ }^{2}$ Department of Pathophysiology, Key Laboratory of State Administration of Traditional Chinese Medicine of thePeople's Republic of China, School of Medicine, Jinan University, Guangzhou, Guangdong, China.

*Corresponding author: Senquan Wu, 873508640@qq.com, Dongguan People's Hospital, 3 Wandao Road South, Dongguan 523059, Guangdong, China; Huadong Wang, owanghd@jnu.edu.cn, Department of Pathophysiology, School of Medicine, Jinan University, Guangzhou 510632, Guangdong, China; Ping Zhang, 1728340667@qq.com, Dongguan People's Hospital, 3 Wandao Road South, Dongguan 523059, Guangdong China.

${ }^{\#}$ These authors have contributed equally to this work. 
1 An efficient scoring model for diagnosis of tuberculous pleural

2 effusion

3

4 ABSTRACT

5 Background: Due to the low efficiency of single clinical feature or laboratory variable

6 in the diagnosis of tuberculous pleural effusion (TBPE), The diagnosis of TBPE is still

7 challenging. This study aimed to build an efficient scoring diagnostic model based on

8 laboratory variables and clinical features to differentiate TBPE from non-tuberculous

9 pleural effusion(non-TBPE).

10 Methods: A retrospective study of 125 patients (63 with TBPE; 62 with non-TBPE)

11 were undertaken. Univariate analysis was used to select the laboratory and clinical

12 variables relevant to the model composition. The statistically different variables were

13 selected to undergo binary logistic regression. Variables B coefficients were used to

14 define a numerical score to calculate a scoring model. A receiver operating

15 characteristic (ROC) curve was used to calculate the best cut-off value and evaluate the

16 performance of the model.

17 Results: Six variables were selected in the scoring model: Age $\leq 46$ years old (4.96

18 point), Male (2.44 point), No cancer (3.19 point), Positive T-cell Spot (T-SPOT) results

19 (4.69 point), Adenosine Deaminase (ADA) $\geq 24.5 \mathrm{U} / \mathrm{L}$ (2.48 point), C-reactive Protein

$20(\mathrm{CRP}) \geq 52.8 \mathrm{mg} / \mathrm{L}$ (1.84 point). With a cut-off value of total score was 11.038 points,

21 the sensitivity, specificity and accuracy of the scoring model was $93.7 \%, 96.8 \%$ and $2299.2 \%$ respectively. 
Conclusion: The scoring model was efficient to differentiate TBPE from non-TBPE.

KEYWORDS: Tuberculosis; Pleural effusion; Tuberculous pleural effusion; Scoring model

\section{Introduction}

Tuberculosis (TB) affects about nine million people and 1.5 million deaths worldwide every year [1]. In 2016, tuberculosis claimed 1.3 million lives among HIV-negative people, exceeding the total number of deaths caused by HIV and becoming the first killer among infectious diseases [2]. On Sep 26, 2018, all UN Member States promised to end the global tuberculosis epidemic by 2030 in the UN General Assembly High Level meeting [3]. Currently, pleural tuberculosis (PT) has been a most common type of extrapulmonary tuberculosis, and its frequency of all TB varies greatly in different countries. PT accounts for more than $20 \%$ of all TB cases in Africa [4, 5], 6.5 to $8.7 \%$ in China [6, 7], 8.7\% in Brazil [8], and 3.7\% in the United States [9]. PT appears to be the main cause of pleural effusion $(\mathrm{PE})$ in countries with a high prevalence of tuberculosis (e.g. in India) [10].

Tuberculous pleural effusion (TBPE) accounts for about 40\% of PE cases in China $[11,12]$. For patients with TBPE, untimely anti-tuberculosis treatment will affect its prognosis. The diagnostic criteria of TBPE is dependent on bacteria culture and histopathology, but the diagnostic sensitivity is limited and time consuming. Thoracoscopic Pleural Biopsy is an effective method in the diagnosis of TBPE, but its application is limited because of its invasiveness, complexity and technical difficulty 
$[5,13,14]$. Moreover, the diagnostic value of single clinical biomarker of TBPE is limited, including erythrocyte sedimentation rate (ESR), blood T-cell spot (T-SPOT), adenosine deaminase (ADA) and lymphocyte ratio, so the diagnosis of TBPE is still challenging. Thus, our study aimed to build an efficient scoring diagnostic model based on laboratory variables and clinical features to differentiate TBPE from non-TBPE.

Methods

\section{Study subjects}

A retrospective study was conducted in the period 2016-2021 after approval by the Ethics Committee of Dongguan People's Hospital. All the enrolled patients met the indications of thoracic puncture or thoracoscopic pleural biopsy and signed the informed contents. The patients selected in the study should meet the following criteria: (1) Adult group; (2) Presence of PE on chest radiographs and ultrasonography; (3) Complete data in clinical were available for all patients. Finally, a total of $125 \mathrm{PE}$ patients were included in this study.

\section{Diagnostic Criteria}

TBPE diagnosis was confirmed when PE met at least one of the following criteria: (1) pleural fluid/sputum/bronchial aspirate/bronchoscopic brushing specimen were positive for acid-fast bacilli or positive culture or positive polymerase chain reaction (PCR) for Mycobacterium tuberculosis. (2) Positive acid-fast staining or epithelioid caseous granuloma in pleural or lung tissue [15-17]. 
Malignant Pleural Effusion (MPE) was diagnosed when pleural histopathology

demonstrated malignant lesions or when cytological examination of pleural effusion demonstrated malignant cells.

Empyema cases with negative M. tuberculosis culture were confirmed according to ATS guidelines [18]. Parapneumonic effusion (PPE) was caused by pneumonia which was confirmed based on the criteria of the American Thoracic Society (ATS) [19]. Thoracoscopic pleural biopsy was performed in patients with unknown etiology of PE. Except for the cases with TBPE, all the other cases were classified as non-TBPE group. All patients underwent a standard thoracocentesis procedure to collect pleural effusion samples and blood was collected by venupuncture before intervention procedures. Record items include: sex, age, clinical symptoms (cough, fever, chest pain, night sweats), T-SPOT, ESR, C-reactive protein (CRP), PE lymphocyte ratio, PE protein, PE lactate dehydrogenase (LDH), ADA, PE location, presence of cancer or not. Patients who had incomplete data were excluded in this study. the statistical analysis was performed using the data from the first pleural fluid sample before treatment. Hematological data were collected from the blood samples taken nearest to the first thoracentesis.

T-SPOT TB test was measured using Enzyme-Linked Immunospot (T-SPOT TB assay kit, Oxford Immunotec Co., Ltd., Abingdon, UK). Pleural effusion protein was measured using Colorimetric Determination (Dry tablets assay kit, Ortho-Clinical Diagnostics Co., Ltd., New York, USA). The activity of ADA was measured using peroxidase assay (ADA assay kit, Beijing Leadman Biochemistry Co., Ltd., Beijing, 
China). LDH levels were measured using the lactic acid substrate method (LDH assay kit, Beckman Coulter Laboratory Systems Co., Ltd., Suzhou, China). CRP level was measured using Quantitative Immunofluorescence method (Boditech Biotechnology Co., Ltd., Guangxi, China). ESR was measured using an Italian

Microsed-System Automatic Blood Sedimentation Instrument. Differential cell counts in PE were counted manually.

\section{Statistical analysis}

The analyses were carried out using SPSS 22.0 software (SPSS Inc., Chicago, IL, USA). Continuous data are reported as median, with first and third quartiles. Mann-Whitney U test was used for comparisons between groups. Chi-square test was used for the analysis of categorical variables. The results with $\mathrm{p}$ values less than 0.05 were considered as statistical significance. Sensitivity, specificity, positive predictive value (PPV), negative predictive value (NPV), accuracy and Youden index were calculated to estimate the diagnostic performance of the indicators. In addition, receiver operating characteristic (ROC) curve was plotted to evaluate the diagnostic value of continuous data for TBPE, and the continuous variable was converted into a binary variable according to the cut-off value corresponding to the maximum value of the Youden Index. The factors with statistically significant differences between the two groups were selected in the binary logistic regression. A goodness-of-fit test of the binary logistic regression model was evaluated by Hosmer and Lemeshow test. Variables B coefficients were used to define a numerical score to calculate a scoring 
model. A receiver operating characteristic (ROC) curve was used to calculate the best cut-off value and evaluate the performance of the scoring model.

\section{Results}

\section{Clinical and laboratory findings of the 125 patients with $P E$}

In this study, a total of 125 patients (63 with TBPE; 62 with non-TBPE) were enrolled according to the selection criteria. The demographic and clinical characteristics were collected from the patient's medical records and summarized in Table 1. It was no significant difference between two groups in cough, chest pain, night sweats, pleural fluid protein and LDH $(p>0.05)$, But there were significant differences among age, male, cancer diagnosis, pleural fluid location, fever, ESR, CRP, T-SPOT, pleural fluid lymphocyte ratio and $\mathrm{ADA}(p<0.05)$. The mean age of TBPE group was lower than that of non-TBPE group. TBPE predominated in men $(46 / 63,73.0 \%)$ who with unilateral pleural effusion $(62 / 63,98.4 \%)$. MPE accounts for most of the non-TBPE $(43 / 62,69.4 \%)$. The proportion of fever, positive T-SPOT and the mean values of ESR, CRP, pleural fluid lymphocyte ratio, ADA in patients with TBPE was higher than that of patients with non-TBPE.

\section{The diagnostic performance of single indicator for TBPE}

ROC curve was used to evaluate the diagnostic value of continuous data (Figure 1A and 1B), and the continuous variable was converted into a binary variable according to the cut-off value corresponding to the maximum value of the Youden Index (Table 
133

134

135

136

137

138

139 under the curve (AUC) value was 0.992 (95\%CI: 0.982-1.000) (figure 2). The

2). The diagnostic performance, including Sensitivity, Specificity, Positive predictive value (PPV), Negative predictive value (NPV) and Accuracy, were calculated and summarized in Table 3.

The multivariate logistic regression of binary variables and the establishment of the scoring model

The factors with statistically significant differences between two groups were selected in the logistic regression, using forward selection method to select indicators to enter the final model. At last, $\mathrm{ESR} \geq 27.5 \mathrm{~mm} / \mathrm{h}$, fever, lymphocyte ratio $\geq 91.5 \%$, unilateral pleural effusion was eliminated by the model, And age $\leq 46.5$ years, male, no cancer, positive T-SPOT, $\mathrm{CRP} \geq 52.8 \mathrm{mg} / \mathrm{L}, \mathrm{ADA} \geq 24.5 \mathrm{U} / \mathrm{L}$ were accepted into the final binary logistic regression model. Hosmer and Lemeshow test Confirmed a good goodness-of-fit test of the binary logistic regression model $(P=0.499)$. Variables B coefficients were used to define a numerical score to calculate a scoring model (table4).

\section{The diagnostic performance of scoring model}

The total score is equal to the sum of the score of the Variables in Table 4 when matching the scoring criteria. The calculation equation as follow: $\mathrm{Y}$ (total score) =Age score + Gender score + Cancer score + T-SPOT score + CRP score + ADA score. ROC curve was used to calculate the best cut-off value. When total score $\geq 11.038$, The area performance of the total scoring model for diagnosis TBPE were Summarized in table 
5.

\section{Discussion}

There are many causes of pleural effusion. Since the high prevalence of TB was occurred in China, TBPE accounts for about $40 \%$ of pleural effusion [11, 12]. Currently, the diagnostic performance of clinical feature or laboratory variable in diagnosing TBPE was poor, therefore it's urgent need to find a new method to diagnose TBPE. Our study had built an efficient scoring diagnostic model by using logistic regression based on laboratory variables and clinical features to differentiate TBPE from non-TBPE.

A total of 15 indicators were included in this study. There were no any symptoms were introduced into the final binary logistic regression model, which shows that clinical symptoms had no significant diagnosis value to the TBPE. And the results was similar to the previously reported data [20]. In terms of pleural effusion tests, unilateral pleural effusion, pleural effusion protein, LDH, and pleural effusion lymphocytes ratio ultimately failed to enter the final model, and it may due to TBPE and MPE are dominated by unilateral lymphocytic exudates [21], and most of the PE in this study were MPE. ESR, a non-specific indicator for the diagnosis of TBPE, as expected, was eventually eliminated by the regression model, even though there was difference in univariate analysis between two groups.

Finally, six indicators of age, sex, cancer, CRP, T-SPOT and ADA were included in the diagnostic scoring model by multivariate binary logistics regression, which showed that those six indicators made a great contribution to the diagnosis. In terms of 
age, the non-TBPE group was significantly older than the TBPE group, which may attribute to the elderly-onset of cancer that was the main causes of non-TBPE. Our result showed that TBPE predominated in men (46/63; 73.0\%), while MPE predominated in females $(37 / 62 ; 59.6 \%)$, and it was similar to the previously reported data of Roberta et al [21]. Consistent with the previously studies [22], we also demonstrated the potential diagnostic value of CRP for TBPE. The sensitivity and specificity of ADA, had a good diagnostic performance in diagnosis of TBPE, were above $90 \%$ in diagnosing TBPE, and T-SPOT also showed a good diagnostic value for the diagnosis of TBPE that the sensitivity and specificity were $93.7 \%$ and $77.4 \%$ respectively. Above results were similar to the previous studies [20, 23, 24]. Hosmer and Lemeshow test confirmed a good goodness-of-fit test of the binary logistic regression model $(p=0.499)$. The performance of the scoring model for diagnosis TBPE were evaluated by ROC cure, when total score $\geq 11.038$, the AUC value was 0.992 (95\%CI: 0.982-1.000), the sensitivity, specificity, PPV, NPV were $93.7 \%, 96.8 \%, 100 \%$ and $93.9 \%$ respectively, the diagnostic performance of the scoring model was better than the reported data of Petborom et al [20, 25]. Our scoring model can aid to diagnosis TBPE and provide more evidence for anti-tuberculosis treatment, but we need to track the effect of treatment to verify the accuracy of the diagnostic model follow-up. When anti-tuberculosis treatment is ineffective, a thoracoscopic pleural biopsy should be used to further confirm the diagnosis. For medical units with limited sanitary conditions, the diagnostic model can help to find TBPE, so that patients can be referred to specialist hospitals for earlier treatment. 

incomplete data were excluded from the study which leading to a reduction in the

201 number of cases. Some test indicators, such as cytokines, CD4 and CD8 were not 202 included in our study because of the small number of people tested. Therefore, the 203 establishment of a better TBPE diagnostic scoring model requires a prospective study, 204 multi-center study to achieve.

\section{Conclusions}

207 The diagnostic score model established by logistic regression combined with multiple 208 indicators improves diagnostic performance. It is significantly higher than a single 209 index of laboratory variables or clinical features in diagnosing TBPE. In brief, 210 establishing a scoring model for the diagnosis of TBPE provide a more economical, 211 effective, and faster diagnostic method based on routine clinical data to assist clinicians 212 in making better clinical decisions.

\section{Abbreviations}

215 TB: Tuberculosis; TBPE: Tuberculous Pleural Effusion; ROC: Receiver Operating 216 Characteristic; T-SPOT: T-cell Spot; ADA: Adenosine Deaminase; CRP: C-reactive Protein; 217 PT: Pleural Tuberculosis; PE: pleural effusion; ESR: Erythrocyte Sedimentation Rate; LDH: 218 Lactate Dehydrogenase; PPV: Positive Predictive Value; NPV: Negative Predictive Value. 
221 Not applicable.

\section{Authors' contributions}

224 All listed authors meet the requirements for authorship. SW, HW and PZ conceived 225 and designed the study. SL, NF and WM collected clinical information. SW, SL and 226 NF analyzed the data. SW wrote the manuscript. All authors critically reviewed and 227 approved the final manuscript.

\section{Funding}

230 This work was supported by grants from the Dongguan Social Science and Technology 231 Development Project [No 202050715001774].

\section{Availability of data and materials}

Most of the data was included in the submission. The more details of the data were

235 available from the corresponding authors on reasonable request.

\section{Declarations}

Ethics approval and consent to participate

240 This study was approved by the Ethics Committee of Dongguan People's Hospital, and 241 the institutional review board approved a waiver of patient informed consent. 
243 Not applicable.

244

\section{Competing interests}

246 No potential conflict of interest was reported by the authors. 


\section{References}

1. Macias A, Sanchez-Montalva A, Salvador F et al: Epidemiology and diagnosis of pleural tuberculosis in a low incidence country with high rate of immigrant population: A retrospective study. Int J Infect Dis. 2019; 78:34-38.

2. Glaziou P, Floyd K, Raviglione MC: Global Epidemiology of Tuberculosis. Semin Respir Crit Care Med. 2018; 39(3):271-285.

3. Harding E: WHO global progress report on tuberculosis elimination. Lancet Respir Med. 2020; 8(1):19.

4. Saks AM, Posner R: Tuberculosis in HIV positive patients in South Africa: a comparative radiological study with HIV negative patients. Clin Radiol. 1992; 46(6):387-390.

5. Zhai K, Lu Y, Shi HZ: Tuberculous pleural effusion. J Thorac Dis. 2016; 8(7):E486-494.

6. Wang X, Yang Z, Fu Y et al: Insight to the Epidemiology and Risk Factors of Extrapulmonary Tuberculosis in Tianjin, China during 2006-2011. PLoS One. 2014; 9(12):e112213.

7. Pang Y, An J, Shu W et al: Epidemiology of Extrapulmonary Tuberculosis among Inpatients, China, 2008-2017. Emerg Infect Dis. 2019; 25(3):457-464.

8. Seiscento M, Vargas FS, Rujula MJ et al: Epidemiological aspects of pleural tuberculosis in the state of Sao Paulo, Brazil (1998-2005). J Bras Pneumol. 2009; 35(6):548-554.

9. Peto HM, Pratt RH, Harrington TA, LoBue PA, Armstrong LR: Epidemiology of extrapulmonary tuberculosis in the United States, 1993-2006. Clin Infect Dis. 2009; 49(9):1350-1357.

10. Udwadia ZF, Sen T: Pleural tuberculosis: an update. Curr Opin Pulm Med. 2010; 16(4):399-406.

11. Villena Garrido V, Cases Viedma E, Fernandez Villar A et al: Recommendations of diagnosis and treatment of pleural effusion. Update. Arch Bronconeumol. 2014; 50(6):235-249.

12. Wang XJ, Yang Y, Wang Z et al: Efficacy and safety of diagnostic thoracoscopy in undiagnosed pleural effusions. Respiration. 2015; 90(3):251255.

13. Steingart KR, Flores LL, Dendukuri N et al: Commercial serological tests for the diagnosis of active pulmonary and extrapulmonary tuberculosis: an updated systematic review and meta-analysis. PLoS Med. 2011; 8(8):e1001062.

14. Wong CF: Early diagnosis of tuberculous pleural effusion: apart from pleural fluid adenosine deaminase, pleural biopsy still has a role. Hong Kong Med J. 2018; 24(3):316-317.

15. Flores-Ibarra AA, Ochoa-Vazquez MD, Sanchez-Tec GA: [Diagnostic strategies in the Tuberculosis Clinic of the Hospital General La Raza National Medical Center]. Rev Med Inst Mex Seguro Soc. 2016; 54(1):122-127.

16. Klimiuk J, Krenke R, Safianowska A, Korczynski P, Chazan R: Diagnostic performance of different pleural fluid biomarkers in tuberculous pleurisy. Adv Exp Med Biol. 2015; 852:21-30. 
17. Abrao FC, de Abreu IR, Miyake DH, Busico MA, Younes RN: Role of adenosine deaminase and the influence of age on the diagnosis of pleural tuberculosis. Int J Tuberc Lung Dis. 2014; 18(11):1363-1369.

18. American Thoracic S, Infectious Diseases Society of A: Guidelines for the management of adults with hospital-acquired, ventilator-associated, and healthcare-associated pneumonia. Am J Respir Crit Care Med. 2005; 171(4):388-416.

19. Niederman MS, Mandell LA, Anzueto A et al: Guidelines for the management of adults with community-acquired pneumonia. Diagnosis, assessment of severity, antimicrobial therapy, and prevention. Am J Respir Crit Care Med. 2001; 163(7):1730-1754.

20. Petborom P, Dechates B, Muangnoi P: Differentiating tuberculous pleuritis from other exudative lymphocytic pleural effusions. Ann Palliat Med. 2020; 9(5):2508-2515.

21. Sales RK, Vargas FS, Capelozzi VL et al: Predictive models for diagnosis of pleural effusions secondary to tuberculosis or cancer. Respirology. 2009; 14(8):1128-1133.

22. Porcel JM, Vives M, Cao G et al: Biomarkers of infection for the differential diagnosis of pleural effusions. Eur Respir J. 2009; 34(6):1383-1389.

23. Zhang M, Li D, Hu ZD, Huang YL: The diagnostic utility of pleural markers for tuberculosis pleural effusion. Ann Transl Med. 2020; 8(9):607.

24. Luo Y, Yan F, Xue Y et al: Diagnostic utility of pleural fluid T-SPOT and interferon-gamma for tuberculous pleurisy: A two-center prospective cohort study in China. Int J Infect Dis. 2020; 99:515-521.

25. Ren $\mathrm{Z}, \mathrm{Hu} \mathrm{Y,Xu} \mathrm{L:} \mathrm{Identifying} \mathrm{tuberculous} \mathrm{pleural} \mathrm{effusion} \mathrm{using} \mathrm{artificial}$ intelligence machine learning algorithms. Respir Res. 2019; 20(1):220. 
Table 1. Clinical and laboratory findings of the 125 patients with PE

\begin{tabular}{|c|c|c|c|c|}
\hline & $\operatorname{Total}(\mathrm{n}=125)$ & TBPE $(n=63)$ & Non-TBPE $(n=62)$ & $\mathrm{p}$ Value \\
\hline Age(years) & $58.0(38.0-70.5)$ & $42.0(29.0-65.0)$ & $67.0(53.0-74.0)$ & 0.000 \\
\hline Male & $71(56.8 \%)$ & $46(73.0 \%)$ & $25(40.3 \%)$ & 0.000 \\
\hline Cancer & $44(35.2 \%)$ & $1(1.6 \%)$ & $43(69.4 \%)$ & 0.000 \\
\hline Unilateral PE & $116(92.8 \%)$ & $62(98.4 \%)$ & $54(87.1 \%)$ & 0.017 \\
\hline Cough & $101(80.8 \%)$ & $52(82.5 \%)$ & $49(79.0 \%)$ & 0.656 \\
\hline Fever & $41(32.8 \%)$ & $33(52.4 \%)$ & $8(12.9 \%)$ & 0.000 \\
\hline Chest pain & $50(40.0 \%)$ & $30(47.6 \%)$ & $20(32.3 \%)$ & 0.080 \\
\hline Night sweats & $4(3.2 \%)$ & $3(4.8 \%)$ & $1(1.6 \%)$ & 0.619 \\
\hline \multicolumn{5}{|l|}{ In blood: } \\
\hline $\operatorname{ESR}(\mathrm{mm} / \mathrm{h})$ & $35.0(21.5-56.0)$ & $40.0(28.0-60.0)$ & $30.0(12.0-45.7)$ & 0.031 \\
\hline $\mathrm{CRP}(\mathrm{mg} / \mathrm{L})$ & $36.1(13.2-90.8)$ & $76.5(30.1-131.3)$ & $18.1(8.4-41.0)$ & 0.000 \\
\hline T-SPOT & $73(58.4 \%)$ & $59(93.7 \%)$ & $14(22.6 \%)$ & 0.000 \\
\hline \multicolumn{5}{|l|}{ In PE: } \\
\hline $\mathrm{L} \%$ & $90(77.5-96.0)$ & $93.0(84.0-98.0)$ & $85.0(67.7-94.0)$ & 0.000 \\
\hline Protein(g/L) & $64.7(53.0-79.6)$ & $69.5(58.5-81.6)$ & $61.7(50.4-77.5)$ & 0.063 \\
\hline $\mathrm{ADA}(\mathrm{U} / \mathrm{L})$ & $26.7(8.6-41.7)$ & $40.9(33.6-49.3)$ & $9.5(7.1-12.9)$ & 0.000 \\
\hline LDH(U/L) & $306.2(216.2-571.4)$ & $415.1(262.0-610.7)$ & $269.4(207.7-392.9)$ & 0.299 \\
\hline
\end{tabular}

Note: Data in the table are expressed either as a frequency (percentage) or a median (interquartile range); PE: pleural effusion; TBPE: tuberculous pleural effusion; nonTBPE: non-tuberculous pleural effusion; ESR: erythrocyte sedimentation rate; CRP: Creactive protein; T-SPOT: T-cell spot; L\%: lymphocyte ratio; $\mathrm{ADA}$ : adenosine deaminase. 
Table 2. Youden Index and cut-off value of continuous data

\begin{tabular}{lll}
\hline & Youden Index & cut-off value \\
\hline Age (years) & 0.443 & 46.5 \\
ESR (mm/h) & 0.23 & 27.5 \\
CRP (mg/L) & 0.457 & 52.8 \\
L\% & 0.264 & 91.5 \\
ADA (U/L) & 0.856 & 24.5 \\
\hline
\end{tabular}

Note: ESR: erythrocyte sedimentation rate; CRP: C-reactive protein; L\%: lymphocyte ratio; $\mathrm{ADA}$ : adenosine deaminase. 
Table 3. The diagnostic performance of single indicator for TBPE

\begin{tabular}{llllll}
\hline & Sensitivity & Specificity & PPV & NPV & Accuracy \\
\hline Age $\leq 46.5$ years & 0.556 & 0.887 & 0.833 & 0.663 & 0.720 \\
Male & 0.730 & 0.597 & 0.648 & 0.685 & 0.664 \\
No cancer & 0.984 & 0.694 & 0.765 & 0.977 & 0.840 \\
Fever & 0.524 & 0.871 & 0.805 & 0.643 & 0.696 \\
Unilateral PE & 0.984 & 0.129 & 0.534 & 0.889 & 0.560 \\
Positive T-SPOT & 0.937 & 0.774 & 0.808 & 0.923 & 0.856 \\
ESR $\geq 27.5 \mathrm{~mm} / \mathrm{h}$ & 0.762 & 0.468 & 0.593 & 0.659 & 0.616 \\
$\mathrm{CRP} \geq 52.8 \mathrm{mg} / \mathrm{L}$ & 0.651 & 0.806 & 0.774 & 0.694 & 0.728 \\
$\mathrm{~L} \% \geq 91.5 \%$ & 0.603 & 0.661 & 0.644 & 0.621 & 0.632 \\
$\mathrm{ADA} \geq 24.5 \mathrm{U} / \mathrm{L}$ & 0.937 & 0.919 & 0.922 & 0.934 & 0.928 \\
\hline
\end{tabular}

Note: PPV: positive predictive value; NPV: negative predictive value; PE: pleural effusion; T-SPOT: T-cell spot; ESR: erythrocyte sedimentation rate; CRP: C-reactive protein; L\%: lymphocyte ratio; ADA: adenosine deaminase. 
Table 4. Score for diagnosis based on the B coefficient of the variables

\begin{tabular}{llcc}
\hline Variable & Scoring criteria & B coefficient & \multicolumn{1}{c}{ Score } \\
\hline Age & Age $\leq 46.5$ years & 4.96 & 4.96 \\
Gender & Male & 2.44 & 2.44 \\
Cancer & No cancer & 3.19 & 3.19 \\
T-SPOT & Positive T-SPOT & 4.69 & 4.69 \\
CRP & CRP $\geq 52.8 \mathrm{mg} / \mathrm{L}$ & 1.84 & 1.84 \\
ADA & ADA $\geq 24.5 \mathrm{U} / \mathrm{L}$ & 2.48 & 2.48 \\
\hline
\end{tabular}

Note: T-SPOT: T-cell spot; CRP: C-reactive protein; ADA: adenosine deaminase. 
Table 5. The performance of scoring model

\begin{tabular}{ll}
\hline & Scoring model \\
\hline cut-off value & $\geq 11.038$ \\
Youden Index & 0.905 \\
Sensitivity & 0.937 \\
Specificity & 0.968 \\
Accuracy & 0.992 \\
PPV & 1.000 \\
NPV & 0.939 \\
\hline
\end{tabular}

Note: PPV: positive predictive value; NPV: negative predictive value. 

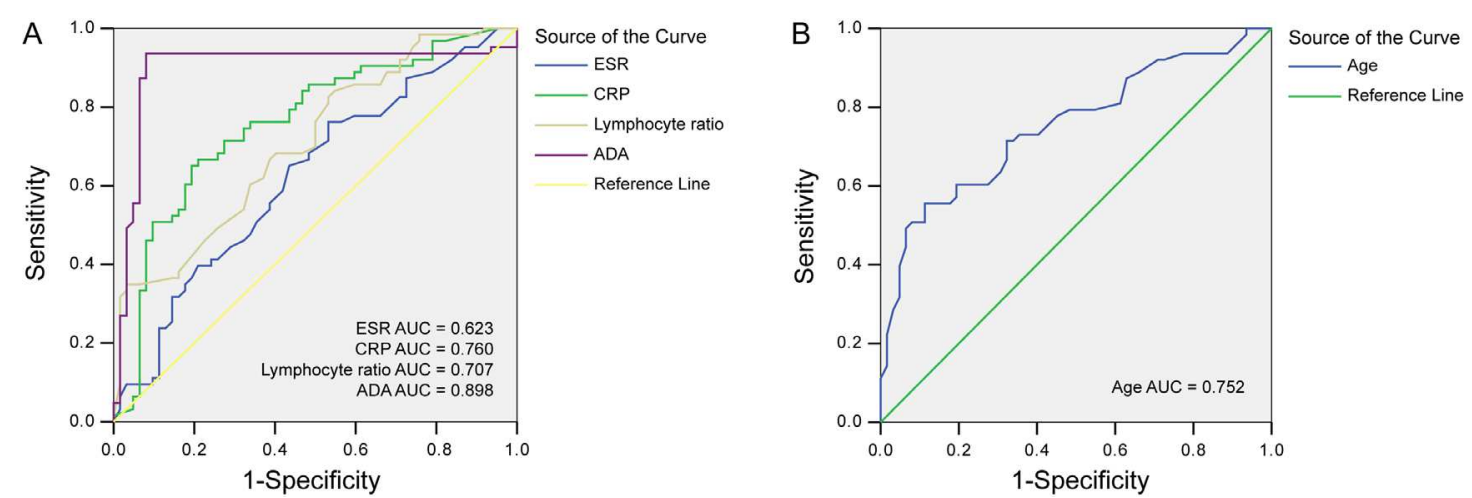

Figure 1. The diagnostic value for TBPE. (A) The diagnostic value of ESR, CRP, Lymphocyte ratio and ADA for TBPE, the AUC value was 0.623, 0.760, 0.707 and 0.898 respectively; AUC: area under the curve. (B) The diagnostic value of Age for TBPE, the AUC value was 0.752 . 


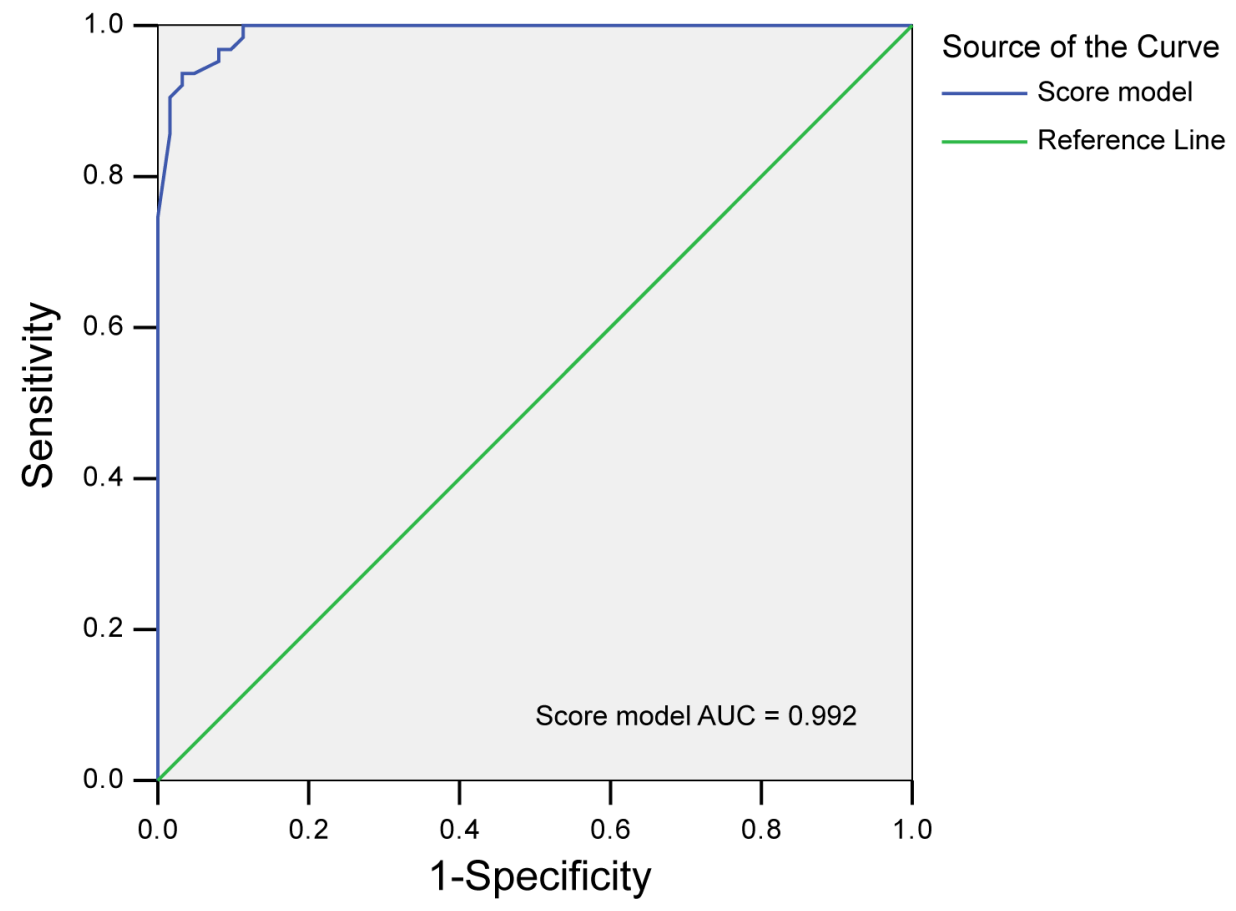

Figure 2. The diagnostic value of Scoring model for TBPE. The AUC value was 0.992; AUC: area under the curve. 